Artigo Original

\title{
Qual é o perfil motivacional característico de tenistas infanto-juvenis brasileiros? ${ }^{1}$
}

\author{
Marcos Alencar Abaide Balbinotti 1,2,3 \\ Luciano Juchem ${ }^{4,6}$ \\ Marcus Levi Lopes Barbosa 5,6 \\ Ricardo Pedrozo Saldanha 5, 6, 7 \\ Carlos Adelar Abaide Balbinotti ${ }^{5,6}$ \\ ${ }^{1}$ Université du Québec à Trois-Rivières, Trois-Rivières, Québec, Canadá \\ ${ }^{2}$ Laboratoire de Méthodes Psychométriques et Expérimentales, \\ Université du Québec à Trois-Rivières, Trois-Rivières, Québec, Canadá \\ ${ }^{3}$ Groupe d'Études en Méthodes psychométriques appliquées à la psychologie du Sport, Université du \\ Québec à Trois-Rivières, Trois-Rivières, Québec, Canadá \\ ${ }^{4}$ Universidade Federal do Vale do São Francisco, Petrolina, PE, Brasil \\ ${ }^{5}$ Universidade Federal do Rio Grande do Sul, Porto Alegre, RS, Brasil \\ ${ }^{6}$ Núcleo de Estudos e Pesquisa em Pedagogia e Psicologia do Esporte (NP ${ }_{3}$ Esporte), Universidade \\ Federal do Rio Grande do Sul, Porto Alegre, RS, Brasil \\ ${ }^{7}$ Faculdade Cenecista de Osório, Osório, RS, Brasil
}

\begin{abstract}
Resumo: Motivação, na Teoria da Autodeterminação (TAD), é entendida como a chave principal do bom funcionamento psicológico do indivíduo e de seu bem-estar geral. De acordo com esta teoria, o "Prazer" é a dimensão que melhor explica o comportamento humano autodeterminado. Este estudo descreve o perfil motivacional característico de tenistas infanto-juvenis brasileiros e explora possíveis diferenças significativas nas médias das dimensões que integram o perfil avaliado pelo Inventário de Motivação à Prática Regular de Atividades Físicas e/ou Esportivas (IMPRAFE-126), controladas pela variável "Sexo". Uma amostra de 226 tenistas gaúchos e catarinenses, com idades entre 13 e 16 anos. Os resultados indicam que a dimensão que mais motiva esses tenistas, independente do sexo, é o Prazer, seguida, respectivamente, pelas dimensões: Competitividade, Saúde, Sociabilidade, Estética e Controle de Estresse. Além de este resultado ser um importante indicador à formulação de modelos de treino, uma das principais conclusões é que este estudo confirma a teoria de base.
\end{abstract}

Palavras-chave: Motivação. Esporte. Tênis. Atletas infanto-juvenis.

\section{What is the motivational profile characteristic of children and young Brazilian players?}

\begin{abstract}
Motivation, in the Self-Determination Theory, is understood as the main key of the individual good psychological functioning and its general well-being. According to this theory, the "Pleasure" is the dimension that better explains the self-determinate human behavior. This study describes the juvenile tennis player motivational profile and explores differences in the average levels of the motivational dimensions that integrate the profile evaluated for the Inventário de Motivação à Prática Regular de Atividades Físicas e/ou Esportivas (IMPRAFE-126), controlled for the variable "Sex". A sample of 226 youthful tennis player (13 to 16 years old), of both sex. The results indicate the dimension that more motivates these tennis player, independent of the sex in analysis, is the Pleasure; followed, respectively, for the Competitiveness, Health, Sociability, Aesthetic and Control of Stress. Beyond this result to be an important aspect to the formulation of models of trainings, one of the main conclusions is that these study it confirms the base theory.
\end{abstract}

Keywords: Motivation. Sport. Junior tennis players.

\section{Introdução}

Este artigo tem como objetivo explorar o perfil motivacional de tenistas infanto-juvenis avaliados pelo Inventário de Motivação à Prática Regular de

\footnotetext{
${ }^{1}$ Artigo derivado de dissertação de mestrado.
}

Atividades Físicas e/ou Esportivas (IMPRAFE126), no contexto da Teoria da Autodeterminação (TAD) de Deci e Ryan (1985; 2002; 2008), conforme a variável de controle "Sexo". Para respondê-lo, apresenta-se o plano teórico relativo à $T A D$ e o modelo de avaliação da motivação 
proposto por Balbinotti (2004). Após a apresentação do método, são calculados os resultados conforme os princípios métricos aceitos na literatura especializada (BALBINOTTI, 2005; PESTANA; GAGEIRO, 2008; REIS, 2008). As discussões têm origem neste processo.

\section{Plano teórico relativo à $T A D$}

Na metade da década de 1970, Deci (1975) apresentou as grandes linhas de sua teoria da motivação humana. Alguns anos mais tarde, Deci (1980) apresentou uma nova versão, um pouco mais elaborada. Entretanto, foi somente na metade da década de 1980 que Deci e Ryan (1985) apresentaram uma versão mais definitiva da Teoria da Motivação Intrínseca e da Autodeterminação no Comportamento Humano (TAD). Menos de 20 anos mais tarde, e com uma base consistente de estudos empíricos realizados, esses autores (RYAN; DECI, 2000a; DECl; RYAN, 2002) apararam importantes arestas teóricas e indicaram direções para futuras pesquisas sobre autodeterminação e motivação (intrínseca, extrínseca e a motivação). Nesta teoria, estar motivado significa estar disposto a agir (RYAN; DECl, 2007). A satisfação das necessidades psicológicas inatas, de competência, de relacionamento com os pares e de autonomia são as bases para 0 desenvolvimento do comportamento automotivado (RYAN; DECl, 2000a; 2000b). Quando autodeterminadas, as pessoas experenciam a liberdade de fazer o que acham interessante, pessoalmente importante $e$ revitalizante (DECl; RYAN, 2008). Apesar do fato de pessoas, quando saudáveis, poderem apresentar um comportamento autodeterminado (motivado) de forma natural, algumas condições são necessárias para a manutenção deste comportamento. Esta teoria procura, então, responder de que forma este comportamento se mantém ou varia, assim como, os fatores pessoais ou dimensões da personalidade que contribuem para este fenômeno (RYAN; DECI, 2007).

\section{Modelo de avaliação da motivação}

Tendo como base a TAD, Balbinotti (2004) elaborou o Inventário de Motivação à Prática Regular de Atividades Físicas e/ou Esportivas (IMPRAFE-126), que, entre outros aspectos, permite avaliar o perfil motivacional de atletas e, a partir de aplicações múltiplas e sucessivas, se o comportamento autodeterminado se mantém, diminui ou até aumenta em função do tempo de prática esportiva. Recentemente ficou demonstrado (BALBINOTTI; BARBOSA, 2008), através de análises fatoriais confirmatórias e cálculos Alpha de Cronbach, que o IMPRAFE-126 avalia com precisão esta motivação, a partir de um grupo de motivos que formam seis dimensões distintas, mas relacionáveis. São elas: "Controle de Estresse" (avalia em que nível as pessoas utilizam o esporte como forma de controlar a ansiedade e o estresse da vida cotidiana); "Saúde" (avalia em que nível pessoas utilizam o esporte como forma de manutenção da saúde geral e de prevenção de doenças associadas ao sedentarismo); "Sociabilidade" (avalia em que nível pessoas utilizam o esporte como forma de fazer parte de um grupo, clube ou relacionar-se com outras pessoas); "Competitividade" (avalia em que nível pessoas utilizam o esporte como forma de manifestação de aspectos relacionados à competição); "Estética" (avalia em que nível pessoas utilizam o esporte como forma de obter ou manter um corpo atraente); e, "Prazer" (Avalia em que nível pessoas utilizam o esporte como forma de obtenção de sensação de bem estar, de diversão e de satisfação que esta prática proporciona). Esse modelo tem sido testado com diferentes esportes e atividades físicas gerais.

\section{Questões centrais da investigação}

Partindo-se dos conteúdos apresentados, foi possível formular as duas seguintes questões centrais desta Investigação: "qual é o perfil motivacional característico dos tenistas infantojuvenis avaliados?" E ainda, "esse perfil permanece o mesmo, independente do sexo dos tenistas em análise?" Para responder estas questões, foram empregados os procedimentos éticos e metodológicos apresentados a seguir.

\section{Material e métodos}

O Comitê de Ética em Pesquisa da Universidade Federal do Rio Grande do Sul analisou e aprovou este projeto (número de referência: 2006569). A escolha da amostra foi por conveniência, evitando-se atletas fora dos critérios de seleção. Os 226 tenistas infantojuvenis, de ambos os sexos (Masculino $=166$; Feminino $=60$ ) e com idades variando de 13 a 16 anos (Média $=14,19 ; \mathrm{DP}=1,05 ;$ Moda $=13$; Mediana $=14$ ), implicados nesta pesquisa eram atletas de competição estadual e/ou nacional, gaúchos e catarinenses. Neste estudo foram utilizados dois instrumentos: um Questionário de 
Identificação das Variáveis de Controle (QIVC) (apenas para controle das variáveis: "Sexo", "Idade" e "se já participaram de Competições Estaduais e/ou Nacionais") e o Inventário de Motivação à Prática Regular de Atividades Físicas e/ou Esportivas (IMPRAFE-126).

O IMPRAFE-126 (BALBINOTTI, 2004; BALBINOTTI; BARBOSA, 2008) é um inventário que avalia seis dimensões associadas à motivação à prática regular de atividades físicas e/ou esportivas: "Controle de Estresse", "Saúde", "Sociabilidade", "Competitividade", "Estética", e "Prazer". Trata-se de 126 itens, agrupados seis a seis, que descrevem motivos que estimulam pessoas à prática regular de um esporte ou atividade(s) física(s). As respostas aos itens do inventário são dadas conforme uma escala bidirecional, de tipo Likert (LIKERT, 1932), graduada em cinco pontos, indo de "isto me motiva pouquíssimo" (1) a "isto me motiva muitíssimo" (5). Os sujeitos levam em média 20 minutos para responder o inventário. Cada dimensão é analisada individualmente. Os dados referentes a sua validade de construto (pelo viés da validade fatorial confirmatória) e fidedignidade (pelo viés da consistência interna) foram apresentados em uma recente publicação (BALBINOTTI, BARBOSA, 2008). Os resultados satisfatórios obtidos garantem seu uso.

\section{Resultados}

Para responder as duas questões desta pesquisa, apresentam-se, sucessiva e sistematicamente, o estudo dos casos extremos e os resultados das estatísticas descritivas gerais e das comparações das médias.

\section{Estudo dos casos extremos}

Foram detectados quatro casos extremos (ver Quadro 1). Todos localizados à esquerda da distribuição dos dados. Tratam-se de 4 casos que puxam para baixo as médias das três dimensões afetadas. Aplicaram-se testes $t$ para uma amostra e seus resultados, independente do sexo e da dimensão em análise $\left[\mathrm{t}_{(58)}<0,41 ; \mathrm{p}>0,70\right]$, indicaram não haver diferenças significativas entre as médias com e sem casos extremos. Sendo assim, decidiu-se manter esses casos, pois na realidade eles existem e podem ser interpretados, à luz da TAD, como amotivados.

Quadro 1. Demonstração Gráfica dos casos extremos, por dimensão em estudo, controlado pela variável "Sexo" ( $M$ = masculino; $F$ = feminino).

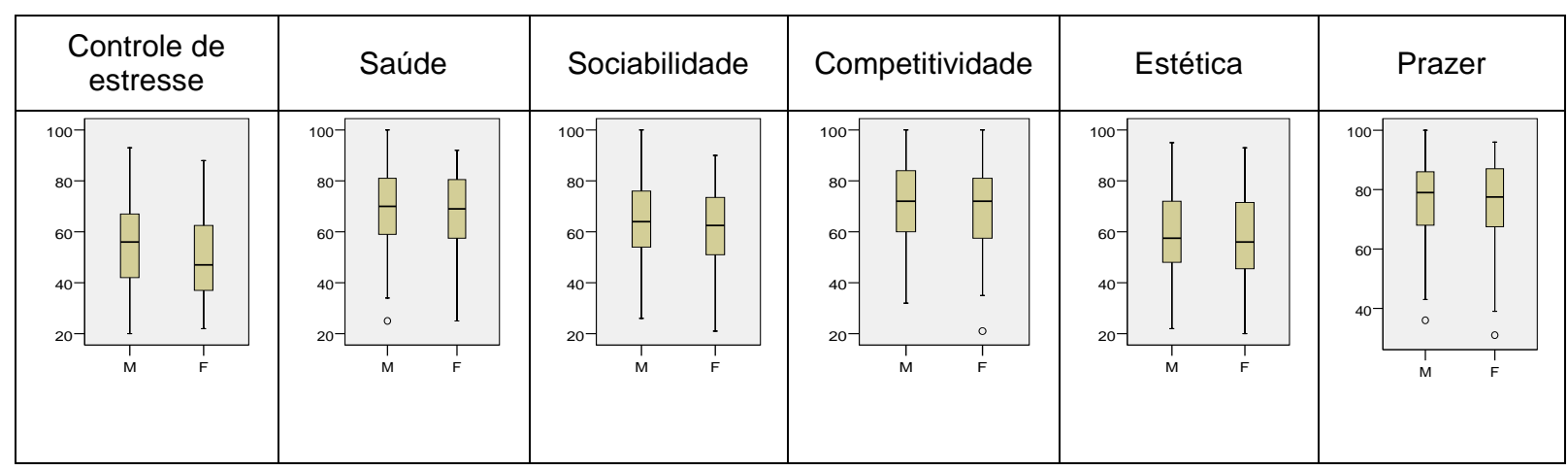

\section{Estatísticas descritivas gerais e das comparações das médias}

Conforme a Quadro 2, destaca-se: a) as médias aritméticas das dimensões motivacionais, independente do controle da variável "Sexo", variaram de 49,4 a 76,7 , deixando tácitas as diferenças nominais encontradas; b) quando controlada a variável Sexo, nota-se diferenças nominais sempre favoráveis aos atletas masculinos, independente da dimensão em análise; c) quando controlada a dimensão e o sexo, nota-se valores semelhantes nas estatísticas de tendência central; e, d) as estatísticas de distribuição garantem a normalidade dos dados em análise, a única exceção ocorreu na simetria da dimensão Prazer com os atletas do sexo masculino.

Os resultados de uma série de testes $t$ para amostras pareadas, quando controlada a variável Sexo, demonstraram: a) as médias da dimensão $\operatorname{Pr}$ são significativamente mais elevadas $[t \geq$ $5,261, p<0,001$ ] que todas as outras; b) as médias das dimensões Co e Sa são estatisticamente iguais $(t=1,216, p=0,225]$, mas são significativamente maiores $[t \geq 6,662, p<$ 0,001] que as das dimensões So, Es e CE; c) a média da dimensão So é estatisticamente maior [t $\geq 4,236, p<0,001$ ] que as médias das dimensões 
Es e CE; e, d) a média da dimensão Es é estatísticamente maior $[t=3,318, p=0,001]$ que a da dimensão CE. Ainda, para explorar o fenômeno das diferenças nominais sempre favoráveis aos atletas masculinos, utilizou-se uma série de testes t para amostras independentes e, após ter-se assumido a igualdade das variâncias $\left[F_{\mathrm{Pr}}=0,453, \mathrm{p}=0,502 ; F_{\mathrm{Co}}=0,331, \mathrm{p}=0,565 ; F_{\mathrm{Sa}}\right.$ $=0,114, p=0,736 ; F_{\mathrm{SO}}=0,147, p=0,701 ; \quad F_{\mathrm{ES}}=$ $\left.0,205, p=0,651 ; \quad F_{C E}=0,024, p=0,876\right]$, comprovou-se que seus resultados $\left[t_{\mathrm{Pr}}=0,653, \mathrm{p}\right.$ $=0,515 ; t_{\mathrm{Co}}=0,808, \mathrm{p}=0,420 ; t_{\mathrm{Sa}}=0,717, \mathrm{p}=$ 0,$472 ; t_{\mathrm{So}}=0,661, \mathrm{p}=0,510 ; t_{\mathrm{Es}}=0,654, \mathrm{p}=$ 0,$\left.514 ; t_{\mathrm{CE}}=2,368, p=0,019\right]$ indicaram que essas diferenças não são significativas. A única excessão ocorreu na dimensão menos motivadora: "Controle de Estresse" (CE), a qual os atletas avaliados manifestam maior motivação que as atletas.

Tabela 1. Dimensões, índices Alpha e estatísticas descritivas relacionadas ao controle da variável Sexo.

\begin{tabular}{|c|c|c|c|c|c|c|c|c|c|}
\hline \multirow{4}{*}{ Dimensões $^{a}$} & \multirow{4}{*}{ 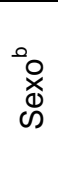 } & \multicolumn{8}{|c|}{ Estatísticas Descritivas } \\
\hline & & \multicolumn{4}{|c|}{ Tendência Central e Dispersãoc $^{c}$} & \multicolumn{4}{|c|}{ Distribuição } \\
\hline & & \multirow{2}{*}{$\bar{X}_{(\mathrm{DP})}$} & \multirow{2}{*}{$\bar{X}_{5 \%}$} & \multirow{2}{*}{$\mathrm{M}_{\mathrm{ed}}$} & \multirow{2}{*}{$M_{\text {od }}$} & \multicolumn{2}{|c|}{ Normalidade } & \multirow{2}{*}{ Ass $^{\dagger}$} & \multirow{2}{*}{$\operatorname{Ach}^{g}$} \\
\hline & & & & & & $K-S^{d}$ & $\underline{\mathrm{p}}$ & & \\
\hline \multirow[t]{2}{*}{ CE } & $\mathrm{M}$ & $55,7_{(17,9)}$ & 55,6 & 56 & 56 & 0,041 & $0,20^{\mathrm{e}}$ & 0,6 & 1,8 \\
\hline & $\mathrm{F}$ & $49,4_{(17,1)}$ & 49,1 & 47 & 48 & 0,101 & 0,19 & 0,7 & 1,3 \\
\hline \multirow[t]{2}{*}{$\mathrm{Sa}$} & M & $69,6_{(15,1)}$ & 69,7 & 70 & 71 & 0,043 & $0,20^{\mathrm{e}}$ & 0,8 & 0,9 \\
\hline & $\mathrm{F}$ & $67,9_{(15,6)}$ & 68,6 & 69 & 63 & 0,070 & $0,20^{\mathrm{e}}$ & 1,2 & 0,3 \\
\hline \multirow[t]{2}{*}{ So } & $M$ & $63,6_{(15,6)}$ & 63,8 & 64 & 64 & 0,047 & $0,20^{\mathrm{e}}$ & 0,9 & 0,8 \\
\hline & $\mathrm{F}$ & $62,1_{(15,7)}$ & 62,4 & 62 & 73 & 0,104 & 0,17 & 1,1 & 0,4 \\
\hline \multirow[t]{2}{*}{ Co } & $M$ & $71,1_{(16,2)}$ & 71,4 & 72 & 73 & 0,058 & $0,20^{\mathrm{e}}$ & 1,2 & 1,7 \\
\hline & $\mathrm{F}$ & $69,1_{(17,3)}$ & 69,6 & 72 & 72 & 0,096 & $0,20^{e}$ & 0,2 & 1,1 \\
\hline \multirow[t]{2}{*}{ Es } & $M$ & $58,9_{(17,4)}$ & 58,9 & 57 & 53 & 0,071 & 0,09 & 0,5 & 1,3 \\
\hline & $\mathrm{F}$ & $57,1_{(18,4)}$ & 57,2 & 56 & 53 & 0,100 & $0,20^{\mathrm{e}}$ & 0,3 & 1,1 \\
\hline \multirow[t]{2}{*}{$\operatorname{Pr}$} & $M$ & $76,7_{(13,4)}$ & 77,2 & 79 & 85 & 0,087 & 0,01 & 2,9 & 0,5 \\
\hline & $\mathrm{F}$ & $75,3_{(14,8)}$ & 76,2 & 77 & 78 & 0,094 & $0,20^{\mathrm{e}}$ & 1,8 & 0,6 \\
\hline
\end{tabular}

${ }^{\text {a }}$ Dimensões: $\mathrm{CE}=$ Controle de Estresse, $\mathrm{Sa}=\mathrm{Saúde}, \mathrm{So}=$ Sociabilidade, $\mathrm{Co}=$ Competitividade, Es $=\mathrm{Estética}, \mathrm{Pr}=$ Prazer;

${ }^{\mathrm{b}} \mathrm{M}=$ Masculino, $\mathrm{F}$ = Feminino;

${ }^{c} \bar{X}(\mathrm{DP})=$ Média Aritmética e Desvio-Padrão; $\bar{X} 5 \%=$ Média Aparada à 5\%; $\mathrm{M}_{\mathrm{ed}}=$ Mediana; $\mathrm{M}_{\mathrm{od}}=\mathrm{Moda}$;

${ }^{d} \mathrm{~K}-\mathrm{S}=$ Kolmogorov-Smirnov com correção Lilliefors,

e Este é o valor mais baixo da verdadeira significância;

${ }^{f}$ Ass = Assimetria (Simetria aceita para valores entre -1,96 e +1,96)

${ }^{g}$ Ach $=$ Achatamento (Curva mesocúrtica aceita para valores entre -1,96 e +1,96)

\section{Discussão}

Os resultados desse estudo permitiram observar os seguintes aspectos:

1) A presença de 4 casos extremos localizados à esquerda da curva de distribuição dos dados, possivelmente implica em casos de atletas que se sentem amotivados, isto é: apresentam um comportamento motivacional percebido em indivíduos que ainda não estão adequadamente aptos a identificar um bom motivo para realizar alguma atividade (RYAN; DECl, 2000a), ao menos nas dimensões motivacionais onde esse fenômeno ocorreu. Poder-se-ia, então, hipotetizar que a dimensão "Saúde" não é suficientemente motivadora para, no mínimo, um sujeito do sexo masculino. Nesta mesma linha, pode-se hipotetizar que o mesmo ocorreu com a dimensão Competitividade (com um sujeito do sexo feminino) e com a dimensão Prazer (com um caso para ambos os sexos). Um estudo futuro, com um design apropriado (pesquisa longitudinal) de acompanhamento dos casos de amotivação, poderia verificar a hipótese de abandono do esporte. Poder-se-ia tentar responder a seguinte questão central: "será que o jovem atleta infantojuvenil, quando avaliado em estado de amotivação em - no mínimo - uma das seis dimensões da motivação, em média, abandona o esporte mais rapidamente que outros que não se encontram neste mesmo estado?" É evidente que o modelo explicativo do comportamento de abandono da prática regular de uma atividade física e/ou esporte é muito mais complexo que uma simples medida, mas será que esta medida não explica uma parte importante da variância explicativa do comportamento de abandono? Questões como estas devem ser rigorosamente respondidas em estudos futuros. 
2) Conforme Urbina (2004), médias nominais já indicam uma tendência de perfil. $E$, de certa forma, o perfil dos atletas representado pelas médias nominais, foi confirmado, quase que absolutamente, por testes estatísticos realizados. Assim, o perfil motivacional característico de tenistas infanto-juvenis brasileiros é: Prazer, Competitividade e Saúde (estatisticamente indiferenciados), Sociabilidade, Estética e Controle de Estresse, nesta ordem. Destaca-se que esse resultado é válido tanto para os atletas do sexo masculino quanto aqueles do sexo feminino. Um estudo recente (BALBINOTTI et al., 2007) com 91 atletas corredores de longa distância, de ambos os sexos e com idades de 14 a 69 anos, demonstrou que o perfil motivacional desses atletas foi diferente para homens $(\mathrm{Pr}, \mathrm{Sa}$, $\mathrm{CE}$, So, Co e Es) e mulheres ( $\mathrm{Pr}$, Sa, CE, Es, So e Co). E outro (BALBINOTTI; CAPOZZOLI, 2008) com 300 praticantes de ginástica de academias do sul do Brasil, de ambos os sexos e com idades de 18 a 65 anos, demonstrou um perfil quase semelhante entre homens ( $\mathrm{Sa}, \mathrm{Pr}$, Es e CE, So e Co) e mulheres (Sa, Pr, Es, CE, So e Co). Este conjunto de resultados de estudos pode estar indicando que cada esporte ou atividade física regular pode ter, em média, um determinado perfil característico. Novos estudos devem testar essa hipótese, pois no caso de se confirmar, essa pode ser uma variável importante a ser explorada quando jovens e/ou adultos estiverem no processo de escolha de um esporte a seguir.

3) Prazer é a que mais motiva esses atletas, independente da variável Sexo. De acordo com Scanlan, Stein e Ravizza (1991), o prazer é a dimensão chave para a compreensão da motivação no desporto. Esse resultado confirma a teoria de base deste estudo, pois, recentemente, Ryan e Deci (2007) afirmaram que o "Prazer" é a dimensão que melhor explica o comportamento humano autodeterminado, diga-se, aquela dimensão que melhor representa a motivação intrínseca, inclusive no contexto do esporte. Posto que esses atletas mostraram comportamentos intrinsecamente motivados, e que esses comportamentos são comumente associados com bem estar psicológico, interesse, alegria e persistência (RYAN; DECl, 2000b), pode-se supor que os atletas avaliados ingressaram neste esporte por vontade própria, diga-se, pelo prazer e satisfação do processo de conhecê-lo, explorálo, aprofundá-lo. Possivelmente, considerando as afirmações de Brière et al. (1995), são atletas que se sentem motivados por experenciar as situações estimulantes inerentes ao esporte escolhido.

4) Observando os resultados da amplitude dos dados (ver Quadro 2), nota-se que o valor mínimo para a dimensão Prazer revela um atleta com o resultado "36" e uma atleta com resultado "31". Essa pequena diferença associada à diferença dos valores da Moda $\left(\mathrm{Mo}_{\mathrm{f}}=78 ; \mathrm{Mo}_{\mathrm{m}}=85\right)$ contribui para a indicação de que os dados em questão não sejam normais. Do ponto de vista puramente estatístico, esse resultado poderia ser interpretado como um problema a ser trabalhado, possivelmente retirando-se o(s) caso(s) aberrante(s) em questão. Em alguns casos resolveria o problema (BALBINOTTI, 2005), mas, de qualquer forma, a decisão de mantê-los já foi tomada e discutida. Entretanto, pode-se interpretar esse resultado de forma positiva, pois está de acordo com a teoria de base deste estudo, como explicado no ponto anterior. Uma das características do atleta profissional é sua autodeterminação. Parece, então, que os atletas infanto-juvenis em análise se comparam aos profissionais e se "autodeterminam" pelo prazer de jogar. Ainda, quando se associa este último resultado àqueles da Mediana $\left(\mathrm{Me}_{\mathrm{f}}=77,5 ; \mathrm{Me}_{\mathrm{m}}=\right.$ 79) e do desvio-padrão $\left(\mathrm{DP}_{\mathrm{f}}=14,8 ; \mathrm{DP}_{\mathrm{m}}=13,4\right)$, independente do sexo em questão, nota-se que esta é a dimensão de maior valor de ponto central e de menor dispersão. Por essas razões não se deve esperar que a distribuição dos dados seja normal, ao menos no caso específico desta dimensão. Ainda, analisando os resultados dos cálculos de distribuição, nota-se que a curva é claramente mesocúrtica (Ach $=0,5)$. O que se encontrou foi que as freqüências de respostas estão localizadas, de forma significativa, em seu lado direto (fenômeno da aquiescência positiva); portanto, o problema da não normalidade estatística está apenas na assimetria dos dados (Ass = 2,9). Assim, pode-se dizer que se trata de uma "deformidade relativa" da distribuição, e não "absoluta". Por fim, assume-se a pertinência dos dados não unicamente por suas bases estatísticas, mas também por sua estrutura teória.

5) Ainda no caso específico da dimensão Prazer, mas agora no sexo feminino, o cálculo Kolmogorov-Smirnov (com correção Lilliefors) revelou que os dados em questão aderem a normalidade estatística $\left(K-S_{(60)}=0,094 ; p>0,05\right)$. Embora possa parecer uma contradição no que acabou de ser dito no ponto anterior, acredita-se 
que o número bem inferior de atletas infantojuvenis do sexo feminino em análise (quando comparado àquele dos atletas do sexo masculino) pode ter influenciado a diferença dos resultados. Além disso, os dois casos extremos encontrados nesta dimensão (um de cada sexo) também pode ter influenciado, efetivamente, nos resultados obtidos. Um estudo pormenorizado com amostras equivalentes (preferencialmente pareadas) seria adequado, mas sabe-se da dificuldade deste procedimento, pois, na realidade, ambas as Federações (Gaúcha e Catarinense) têm um número bem maior de atletas do sexo masculino inscritos. Sabe-se que cerca de $25 \%$ dos inscritos nestas federações são do sexo feminino. No mais, parece que, de maneira geral, nossa cultura tende a apoiar mais os atletas profissionais de sexo masculino (seja em função do prêmio em dinheiro, seja em função do investimento na estrutura dos espetáculos esportivos, etc.). Esta pode ser uma explicação para o estilo de resposta mais positiva dos atletas infanto-juvenis do sexo masculino. Será que eles tendem a responder mais positivamente que as atletas em função de pensarem ter uma expectativa mais concreta e financeiramente mais estável de carreira futura? Não se pode afirmar com certeza que esta seja a razão principal para esta diferença, mas sugerimos estudos subsequentes que expliquem (explorem) estas e outras possibilidades.

6) Ficou demonstrado que a ordem das dimensões não apresenta diferença, diga-se, 0 perfil motivacional característico desses atletas, independente do sexo, é o mesmo. Entretanto, todos os valores nominais das médias são superiores nos atletas do sexo masculino. Mesmo que as diferenças nas médias não tenham sido significativas, existe uma tendência implícita que merece uma atenção especial. Mais uma vez, parece válido a explicação supracitada, a qual indica que nossa cultura provavelmente apoia mais atletas do sexo masculino. Mas também é importante referir que, de acordo com vários autores (WHITE; DUDA, 1994; NEWTON; DUDA; 1993; FORTIER et al. 1995; PETHERICK; WEIGAND, 2002), os homens possuem maior interesse por demonstrar competência em situações esportivas, e esta característica pode explicar, ao menos em parte, esta diferença entre os valores nominais das médias.

7) Novos estudos devem ser conduzidos para que este modelo possa ser testado em diferentes esportes. Trata-se de um inventário promissor e que parece ser suficiente para discriminar um perfil motivacional característico para cada esporte ou atividade física regular. Além disso, recomenda-se a realização de estudos transculturais para que se possa testar a hipótese da semelhança do perfil característico de um esporte, independente da cultura ou país de origem dos atletas.

\section{Referências}

\section{BALBINOTTI, M.A.A. Inventário de Motivação à Prática Regular de Atividades Físicas e/ou Esportivas. Porto Alegre: Núcleo de Estudos de Pedagogia e Psicologia do Esporte da Universidade Federal do Rio Grande do Sul, 2004.}

BALBINOTTI, M.A.A. Para se avaliar o que se espera: reflexões acerca da validade dos testes psicológicos. Aletheia, Canoas, n.21, p.43-52, 2005.

BALBINOTTI, M.A.A.; CAPOZZOLI, C.J. Motivação à prática regular de atividade física: um estudo exploratório com praticantes em academias de ginástica. Revista Brasileira de Educação Física e Esporte, São Paulo, n.22, p.63-80, 2008.

BALBINOTTI, M.A.A.; BARBOSA, M.L.L. Análise da consistência interna e fatorial confirmatório do IMPRAFE-126 com praticantes de atividades físicas. Revista PSICO-USF, Bragança Paulista, n.13, p.43-59, 2008.

BALBINOTTI, M.A.A. et al. Dimensões Motivacionais de Atletas Corredores de Longa Distância: Um Estudo Descritivo-Comparativo Segundo o Sexo. Coleção Pesquisa em

Educação Física, Jundiaí, v. 6, n. 2, p. 73-80, set. 2007. Disponível em:

$<$ http://www.editorafontoura.com.br/loja63/produto $\mathrm{s}$ descricao.asp?lang $=\mathrm{pt}$ BR\&codigo produto $=40$ >. Acesso em: 14 abr. 2010.

BRIÈRE, N.; VALLERAND, R.; BLAIS, M.; PELLETIER, L. Développement et validation d'une mesure de motivation intrinsèque, extrinsèque et d'amotivation en contexte sportif: l'échelle de motivation dans les sports. International Journal of Sport Psychology, Roma, n.26, p.465-489, 1995.

DECI, E.L. Intrinsic motivation. New York: Plenum Publishing Co., 1975.

DECI, E.L.The psychology of selfdetermination. Lexington, Massachusetts: D. C. Heath (Lexington Books), 1980. 
DECI, E.L.; RYAN, R.M. Intrinsic Motivation and self-determination in human behavior. New York: Plenum, 1985.

DECI, E.L.; RYAN, R.M. Self-determination research: Reflections and future directions. In: DECI, E.L.; RYAN, R.M (Eds.). Handbook of selfdetermination research, Rochester, NY: University of Rochester Press, 2002, p.431-441.

DECI, E.L.; RYAN, R.M. Self-Determination Theory: an approach to human motivation and personality. 2008. Disponível em: <http://www.psych.rochester.edu/SDT/index.html> . Acesso em: 09 set. 2008.

FORTIER, M.S.; VALLERAND, R.J.; BRIÈRE, N.M.; PROVENCHER, P.J. Competitive and recreational sport structures and gender: A test of their relationship with sport motivation.

International Journal of Sport Psychology, Roma, n.26, p.24-39, 1995.

LIKERT, R. A Technique for the Measurement of Attitudes". Archives of psychology, New York, n.140, p.1-55, 1932.

NEWTON, M.; DUDA, J.L. Elite Adolescent Athletes' Achievement Goals and Beliefs Concerning Success in Tennis. Journal of Sport e Exercise Psychology, Champaign, n.15, p.437448, 1993.

PESTANA, M.H.; GAGEIRO, J.G. Análise de dados para ciências sociais: a

complementaridade do SPSS. 5aㅡ. Ed., Lisboa: Silabo, 2008.

REIS, E. Estatística descritiva. Lisboa: Silabo, 2008.

PETHERICK, C.M.; WEIGAND, D.A. The Relationship of Dispositional Goal Orientations and Perceived Motivational Climates on Indices of Motivation in Male and Female Swimmers. International Journal of Sport Psychology, Roma, n.33, p.218-237, 2002.

RYAN, R.M.; DECI, E.L. Intrinsic and Extrinsic Motivations: Classic Definitions and New Directions. Contemporary Educational Psychology, New York, n.25, p.54-67, 2000a.

RYAN, R.M.; DECI, E.L. Self-Determination Theory and the Facilitation of Intrinsic Motivation, Social Development, and Well Being. American Psychologist, Washington, n.55, p.68-78, 2000b.

RYAN, R.M.; DECI, E.L. Active Human Nature: Self-Determination theory and the promotion and maintenance of sport, exercise, and health. In: HAGGER, M.S.; CHATZISARANTIS, N.L.D. (eds.). Intrinsic Motivation and Self-
Determination in Exercise and Sport. Human Kinetics, p.1-19, 2007.

SCANLAN, T.K.; STEIN, G.L.; RAVIZZA, K. An in-depth study of former élite figure-skaters: III. Sources of stress. Journal of Sport and Exercise Psychology, Champaign, n.13, p.103120, 1991.

URBINA, S. Essentials of Psychological Testing. Florida: John Wiley \& Sons, 2004.

WHITE, S.A.; DUDA, J.L. The relationship of gender, level of sport involvement, and participation motivation to task and ego orientation. International Journal of Sport Psychology, Roma, n.25, p.4-18, 1994.

\section{Endereço:}

Ricardo Pedrozo Saldanha R. Orfanotrófio, 600 Bloco 11/211Bairro: Cristal Porto Alegre RS Brasil 908440-440

e-mail: ricardo@ricardosaldanha.com.br

Recebido em: 18 de outubro de 2011.

Aceito em: 11 de novembro de 2012.

Motriz. Revista de Educação Física. UNESP, Rio Claro, SP, Brasil - elSSN: 1980-6574 - está licenciada sob Creative Commons - Atribuicão 3.0 